\title{
Percepção dos formandos do Centro de Ciências da Saúde da Universidade Federal de Santa Catarina sobre o que é promoção da saúde
}

\author{
Laís Olsson*, Ana Clara Loch Padilha*, Dayane Machado Ribeiro** \\ * Acadêmica do curso de Odontologia, Universidade Federal de \\ Santa Catarina (UFSC) \\ ** Doutora em Odontologia em Saúde Coletiva. Mestre em \\ Odontologia em Saúde Coletiva. Especialista em Endodontia. \\ Cirurgiã-dentista. Profa. Adjunto III, Universidade Federal de Santa \\ Catarina (UFSC)
}

\section{RESUMO}

Como resposta aos desafios sanitários contemporâneos, a Promoção da Saúde delineia-se num campo teórico-prático-político envolvendo ações e projetos em saúde, apresentando-se em todos os níveis de complexidade da gestão e atenção do sistema de saúde. Para tanto, deve incluir capacitação da comunidade para que a mesma, visando melhoria de qualidade de vida e saúde, possa atuar juntamente no controle desse processo. Dada a importância desse conhecimento, o presente trabalho propô-se identificar a percepção dos formandos do Centro de Ciências da Saúde (CCS) da Universidade Federal de Santa Catarina (UFSC) sobre o que é Promoção da Saúde. Metodologia: uma pesquisa de campo com caráter descritivo e qualitativo visando analisar a realidade a partir da observação de fenômenos e causas. Os sujeitos da pesquisa foram compostos por formandos dos cursos do CCS da UFSC, sendo 3 representantes de cada curso, em função da saturação de respostas, totalizando 15. As informações pessoais foram submetidas a uma análise descritiva, bem como qualitativa através do processo de Análise-Reflexão-Síntese. Resultados: O perfil da amostra foi predominantemente feminino $(60 \%)$, com aproximadamente 21 anos de idade. Quanto ao conhecimento sobre a Promoção da Saúde, observou-se dificuldade em conceituar seu significado, sendo que para a maioria da amostra não foi possível diferenciar prevenção de promoção da saúde, além da inca- pacidade de exemplificar como aplicar em sua prática profissional. Apesar da importância da Promoção da Saúde ter sido reconhecida e exaltada, pôde-se observar falta de conhecimento e entendimento sobre o tema.

\section{DESCRITORES}

Promoção de Saúde. Saúde Bucal. Ensino.

- romoção da saúde é o nome dado ao processo de capacitação da comunidade para atuar na melhoria de sua qualidade de vida e saúde, incluindo uma maior participação no controle deste processo. ${ }^{1}$ Para Campos et al. (2004), a promoção da saúde é um campo teórico-prático-político que em sua composição com os conceitos e as Posições do Movimento de Reforma Sanitária delineia-se como uma política que deve percorrer o conjunto das ações e projetos em saúde, apresentando-se em todos os níveis de complexidade da gestão e da atenção do sistema de saúde. ${ }^{2}$ Assim, trata-se de uma resposta aos desafios sanitários contemporâneos, surgida nos anos 70, como proposta que assume o status de uma das principais linhas de atuação da Organização Mundial da Saúde (OMS) influenciando nos anos seguintes, a elaboração de políticas de saúde de diversos países. ${ }^{3}$ Sendo assim, a Promoção da Saúde não se dirige a uma determinada doença ou desordem, mas serve para aumentar a saúde e o bem estar gerais. ${ }^{4}$ 
Stotz \& Araújo (2004) afirmam que profissionais e técnicos são educadores, apesar de não ter consciência desse papel. Desta forma, faz-se necessário pensar na educação desses educadores no contexto de novas práticas de saúde. ${ }^{5}$

Numa avaliação da reformulação curricular do curso de Medicina da Universidade Federal Fluminense (UFF), Koifman (2001) concluiu ser necessária a introdução de conteúdos da área das ciências sociais, como instrumento para a formação do médico capaz de atuar nos problemas de saúde mais recorrentes da população brasileira, bem como no domínio das tecnologias. O médico deve estar apto a questionar seu papel e a atuar no sentido de melhorar a qualidade de vida e de reduzir os níveis do adoecer.

Botazzo (2003) faz uma crítica sobre o ensino da Odontologia, que tem se baseado em conteúdos técnicos fortemente arraigados ao ambulatório, e a pouca discussão das abordagens sociais dos problemas de saúde entre os estudantes e professores. ${ }^{7}$ Num debate sobre a formação universitária, Araújo (2006) afirma que é mais importante aprofundar os princípios sobre o tipo de saúde que está sendo oferecido à coletividade à abordagem das referências da saúde coletiva. Dessa forma, a formação universitária deve ultrapassar o campo da saúde bucal coletiva, estendendo-se para todas as áreas, acabando com as dicotomias entre básico e clínico, entre clínico e social e entre público e acadêmico. Assim, compete ao ensino superior a formação de profissionais capazes de atuar nesse novo contexto, conduzindo, de maneira contínua, em direção a uma formação integral. ${ }^{8}$

Diante do exposto, a escolha deste tema baseia-se na escassez de publicações que avaliam o entendimento dos formandos quanto a Promoção da Saúde. Conforme a Constituição Federal, em seu artigo 196, a saúde é um direito de todos e dever do Estado. Por sua vez, a Promoção da Saúde atua sobre os determinantes da saúde para criar o maior benefício para a população, contribuindo de maneira significativa para a redução das iniquidades em questão de saúde, desta forma, assegurando os direitos humanos.

Dada a importância do conhecimento sobre a Promoção da Saúde, evidencia-se também a necessidade de identificar o grau de conhecimento dos formandos da área da saúde desta Instituição, o que possibilitará uma discussão quanto ao enfoque dado pelos cursos de graduação. Acreditando ser este conhecimento uma lacuna no ensino da graduação dos estudantes da área da saúde, o presente trabalho propô-se a identificar a percepção dos formandos do Centro de
Ciências da Saúde da UFSC sobre o que é Promoção da Saúde.

\section{METODOLOGIA}

O presente estudo transversal caracteriza-se como uma pesquisa de campo, de caráter descritivo e qualitativo, que teve como objetivo analisar a realidade a partir da observação de fenômenos e causas, após aprovação do Comitê de Ética em Pesquisa (parecer consubstanciado do projeto 152/2007).

A população do estudo foi composta por formandos do Centro de Ciências da Saúde (CCS) da Universidade Federal de Santa Catarina (UFSC), sendo 3 alunos de cada um dos cursos do CCS: Nutrição, Enfermagem, Odontologia, Medicina e Farmácia, totalizando 15 alunos, selecionados de forma aleatória, e em função da saturação de respostas.

Os dados foram obtidos através de um formulário, elaborado e aplicado pelos próprios pesquisadores, composto de questões relacionadas à identificação do entrevistado e de uma pergunta sobre Promoção da Saúde, sendo a resposta gravada e transcrita para posterior análise.

As informações pessoais foram submetidas a uma análise descritiva conforme característica dos dados. Com relação à questão aberta, os dados foram analisados, a partir da primeira entrevista, pelo processo de Análise-Reflexão-Síntese. ${ }^{9}$ Nesse processo a análise decompõe os dados, a síntese os integra às diversas dimensões e contexto da vida dos sujeitos. A análise e a síntese são realizadas de maneira sinérgica através da reflexão, que é uma consideração de dados, associando sensibilidade e razão.

Partindo dessas considerações, o tema proposto é apresentado a partir de dados empíricos relativos a depoimentos selecionados do conjunto de dados dos participantes do estudo e analisados com apoio da literatura.

\section{RESULTADOS E DISCUSSÃO}

O perfil da amostra estudada pode ser observado na Tabela 1.

Pôde-se observar que o gênero predominante foi o feminino (60\%), demonstrando uma maior atuação deste gênero nas áreas relacionadas à saúde. Quanto à idade dos participantes, aproximadamente $25 \%$ dos mesmos estava se formando com apenas 21 anos, sendo a idade mais encontrada na amostra.

O entendimento de promoção da saúde é observado em algumas declarações, como a que traz o Ministério da Saúde, como sendo: 
Tabela 1 - Perfil da amostra composta por formandos dos cursos de graduação do CCS da UFSC.

\begin{tabular}{|c|c|c|c|}
\hline Participante & Sexo & Idade & Especialidade pretendida \\
\hline E.01 & fem. & 26 & Pediatria \\
\hline E.02 & fem. & 24 & Saúde pública \\
\hline E.03 & fem. & 21 & Adm. em enfermagem \\
\hline F.01 & fem. & 21 & Atenção Básica \\
\hline F.02 & fem. & 30 & Análises Clínicas \\
\hline F.03 & fem. & 21 & Análises Clínicas \\
\hline M.01 & masc. & 24 & Radiologia \\
\hline M.02 & masc. & 26 & Endocrinologia \\
\hline M.03 & fem. & 26 & Psiquiatria \\
\hline N.01 & fem. & 24 & Nutrição Clínica \\
\hline N.02 & masc. & 28 & Não sabe \\
\hline N.03 & fem. & 23 & Nutrição Clínica \\
\hline O.01 & masc. & 25 & Cirurgia Bucomaxilofacial \\
\hline O.02 & masc. & 22 & Prótese \\
\hline O.03 & masc. & 21 & Ortodontia \\
\hline
\end{tabular}

Uma estratégia de articulação transversal na qual se confere visibilidade aos fatores que colocam a saúde da população em risco e às diferenças entre necessidades, territórios e culturas presentes no nosso país, visando à criação de mecanismos que reduzam as situações de vulnerabilidade, defendam radicalmente a equidade e incorporem a participação e o controle sociais na gestão das políticas públicas. ${ }^{10}$

Essa visão holística da Promoção da Saúde pôde ser observada em declarações de alguns participantes, que expressam como

"independente de práticas e tratamentos ligados a definidas áreas de saúde... ajude a pessoa a ter um sentido amplo de bem estar" (M.03).

Esse entendimento, contudo, é de difícil compreensão, como foi relatado em outras entrevistas:

"É pergunta difícil." (M.01)

ou ainda

"o problema é a pratica, né, acho que aqui no nosso centro a gente consegue ter mais contato porque lidamos com isso... mas nos outros centros, eu acho que as pessoas não tem noção do que é promoção da saúde... as pessoas acham que isso é de pobre." (F.01), concordando com Alves et al. (1996), que destaca a dificuldade do consenso a respeito de uma definição operacional para esse termo. ${ }^{11}$

Embora, o modelo inicial de promoção da saúde proposto por Leavell \& Clark, tivesse a Promoção da Saúde como um dos elementos do nível primário na medicina preventiva, ${ }^{4}$ seu significado foi reformulado e atualizado, trazendo um maior enfoque político e técnico sobre este tema. Entretanto, o aspecto da prevenção ainda foi bastante citado na definição do tema, como se observa em:

"É o âmbito de tu tentar, através de ações preventivas, promover a saúde." (O.02),

ou:

"É tu atender o paciente de uma forma que tu promova a prevenção.” (O.01),

da mesma forma trazido como:

"Promoção de saúde pra mim é tudo aquilo que pode ser feito para prevenir alguma enfermidade, [...] tudo que age na prevenção seria o mais ideal, né.” (N.02).

Segundo Sícoli \& Nascimento (2003), persistem controvérsias na definição da promoção da saúde e confusões relativas a seus limites conceituais com a prevenção. ${ }^{12}$ Já Acosta \& Duarte (2007), afirmam haver diferenças claras entre as ações de Prevenção e Promoção, e concordam que existe sim, certa confusão sobre o tema. ${ }^{13}$

A prevenção visa à modificação de comportamento individual, reduzindo riscos para determinadas doenças, com maior enfoque clínico; já a Promoção da Saúde vem como uma estratégia de intervenção entre pessoas e o meio ambiente juntando escolhas individuais com responsabilidade social pela saúde, propondo a participação da população. ${ }^{13}$

A Saúde como sinônimo de ausência de doença também foi relatado durante as entrevistas enquanto a definição de Promoção da Saúde era exposta:

\footnotetext{
"são estratégias que a gente tem pra fazer pra pessoa não ficar doente" (N.03).
}

Entretanto, a mudança paradigmática que traz a Promoção da Saúde sugere que em um estado saudável a ausência de doença não é suficiente, nem mesmo necessária. ${ }^{14}$ 
A Saúde Pública, principalmente no contexto da atenção básica foi citada como responsável principal pela Promoção da Saúde, observada, por exemplo, na definição:

"São as estratégias aplicadas nas unidades da atenção básicas de saúde" (M.01).

Contudo, a promoção da saúde delineia-se como uma política que deve percorrer o conjunto das ações e projetos em saúde, apresentando-se em todos os níveis de complexidade da gestão e da atenção do sistema de saúde. ${ }^{15}$

Como público alvo das estratégias de promoção da saúde, observa-se um direcionamento para uma população de mais baixa renda, como citado, por exemplo em

"dar atenção pros pobres, principalmente que não tem acesso" (F.03),

e ainda comentado por outro participante:

"as pessoas acham que isso é de pobre" (F.01).

As áreas abrangidas nesse processo ficaram restritas ao campo da saúde, conforme encontrado em um grande número de entrevistas, que expuseram como envolvidas apenas

“as áreas da saúde” (F.02, O.02, N.01).

A ocorrência da Promoção da Saúde, entretanto, ultrapassa a assistência clínica e propõe ações intersetoriais, que incluem educação, saneamento básico, habitação, renda, trabalho, alimentação, meio ambiente, lazer, entre outros. ${ }^{12}$

A relevância da educação foi relatada em muitas das entrevistas, e definida como

"uma troca" (E.01)

ou ainda,

"um processo dinâmico... todo mundo ensina e aprende

ao mesmo tempo, né?”(M.02)

e a justificativa da mesma foi descrita por alguns participantes pela necessidade de

"se conhecer aquilo que se ta fazendo" (M.03) pois,

"quando a pessoa não entende o porque das coisas, dificilmente ela faz" (N.01).

A população alvo para a educação descrita por parte dos entrevistados demonstrou interesse pelas crianças:

"O educado sempre parte da infância, sempre a primeira infância" (F.02),

citado ainda em exemplos de aplicabilidade como

"vídeos para crianças" (O.02).

Enquanto a meta da educação em saúde visa tornar os indivíduos melhores equipados internamente para que possam fazer escolhas mais saudáveis, a promoção da saúde tenta fazer com que essas escolhas mais saudáveis tornem-se também mais fáceis. Para isso, tenta modificar as normas da sociedade e do meio ambiente afim de que se torne mais favoráveis a obtenção de saúde. ${ }^{14}$

Pode-se observar uma dificuldade por parte dos participantes de aplicabilidade da Promoção da Saúde na área de sua escolha para atuação, como observamos na negação de qualquer possibilidade em

"não, a prótese dentária já é [...] digamos que a última área, [...] é a área que tu vai reabilitar o paciente" (O.02),

ainda visto em

“no hospital não é nem um pouco aplicável, o paciente entra, a gente cuida dele ali rapidinho, ele sai e fica por isso mesmo" (N.01)

e em

"a radiologia odontológica é muito voltada para diagnóstico... então é... não entra muito promoção da saúde” (M.01).

Sobre a importância da Promoção da Saúde, na unanimidade das entrevistas, ela foi positiva, entretanto, um descaso da universidade foi observado, como exposto em

"eu acho que não é dado, eu acho que nosso curso ele ainda continua sendo muito técnico, a gente sai muito 
Percepção dos formandos do Centro de Ciências da Saúde da Universidade Federal de Santa Catarina sobre o que é promoção da saúde • Olsson L, Padilha ACL, Ribeiro DM

despreparado da faculdade" (O.01)

e em

"existe ainda, alguns contrastes... até porque é... a geração que se formou anterior, que teve uma educação diferente é quem ta nos ensinando, então quer dizer, eles primeiro tem que mudar o seu... o seu... seu estilo, a sua consciência pra transmitir pra esse pessoal que ta vindo novo aî" (M.02).

Segundo Botazzo (2003), o ensino odontológico tem se baseado em conteúdos técnicos fortemente arraigados ao ambulatório, e a pouca discussão das abordagens sociais dos problemas de saúde entre os estudantes e professores. ${ }^{7}$

\section{CONCLUSÕES}

Os dados analisados permitiram concluir:

- O perfil da amostra composta por formandos do Centro de Ciências da Saúde da UFSC foi predominantemente feminino $(60 \%)$, com aproximadamente 21 anos de idade.

- Quanto ao conhecimento sobre a Promoção de Saúde, os formandos demonstraram enorme dificuldade em conceituar, definir ou explicar seu significado e quase que total falta de noção de como aplicar em sua prática profissional diária. Para a maioria dos entrevistados não foi possível diferenciar prevenção de promoção da saúde, tendo sido inclusive utilizado a prevenção como definição para promoção.

- Apesar da importância da Promoção da Saúde ter sido reconhecida e exaltada, pôde-se observar falta de conhecimento e entendimento sobre o tema.

\section{ABSTRACT}

The perception of senior students from the Health Science Center at the Federal University of Santa Catarina regarding health promotion

In response to the current health challenges, Health Promotion deals with a theoretical-practicalpolitical approach, involves actions and health projects, and underlies all levels of complexity of health system management and of healthcare service provision. It must prepare the community to be able to help in supervising the whole process to improve the quality of life and health. Given the importance of this knowledge, this paper intends to show how senior students from the Health Science Center at the
Federal University of Santa Catarina (HSC-FUSC) perceive Health Promotion. Methodology: Field research in the form of a descriptive and qualitative survey, aiming at analyzing the real situation by observing different phenomena and their causes. The survey respondents consisted of senior undergraduate students from HSC-FUSC, specifically, 3 students from each course, resulting in 15 students. The personal information was submitted to a descriptive analysis, as well as to qualitative analysis using the process of Analysis-Reflection-Synthesis. Results: The profile of the surveyed respondents was predominantly female $(60 \%)$, and the average age was 21 years old. In respect to the students' knowledge of Health Promotion, they demonstrated difficulty in grasping its meaning; most of the respondents could not distinguish health prevention from health promotion, and were also unable to exemplify how they would apply it in their professional practice. Although the importance of Health Promotion is indeed recognized and valued, a lack of understanding and knowledge of this subject prevailed.

\section{DESCRIPTORS}

Health Promotion. Oral Health. Teaching. -

\section{REFERÊNCIAS}

1. Organização Pan-americana da Saúde. Carta de Ottawa: Ottawa, novembro de 1986. Primeira Conferência Internacional Sobre Promoção da Saúde [documento na internet]. [Acesso em 03 jun 2010]. Disponível em: http://www.opas.org.br/ promocao/uploadArq/Ottawa.pdf. Acesso em: 03 jun. 2010.

2. Campos GW, Barros RB de, Castro AM de. Avaliação de política Nacional de Saúde. Ciên Saúde Colet. 2004; 9(3):745-749.

3. Carvalho SR. Os múltiplos sentidos da categoria "empowerment" no projeto de promoção de saúde. Cad Saúde Pública, 2004; 20(4):1088-1095.

4. Leavell H, Clark G. Medicina preventiva. São Paulo: Mcgraw-hill do Brasil, 1976.

5. Stotz EM, Araujo JWG. Promoção da saúde e cultura política: a reconstrução do consenso. Saúde Soc, 2004; 13(2):5-19.

6. Koifman L. O modelo biomédico e a reformulação do currículo médico da Universidade Federal Fluminense. Hist Ciênc Saúde - Manguinhos, 2001; 8(1):48-70.

7. Botazzo C. Saúde bucal e cidadania: transitando entre a teoria e a pratica. In: Pereira AC, orgnizador. Odontologia em saúde coletiva: planejando ações e promovendo saúde. Porto Alegre: Editora Artmed; 2003. p.17-27.

8. Araujo ME de. Palavras de silêncio na Educação superior em Odontologia. Ciên Saúde Colet, 2006; 11(1):79-182.

9. MINAYO, Maria Cecília de Souza. O desafio do conhecimen- 
to: pesquisa qualitativa em saúde. 8. ed. São Paulo: Hucitec, 2004.

10. Brasil. Ministério da Saúde. Política Nacional de Promoção da Saúde [documento na internet]. [acesso em 03 jun 2010]. Disponível em: http://bvsms.saude.gov.br/bvs/publicacoes/ portaria687_2006_anexo1.pdf

11. Alves ED, Arratia A, Silva DG da. Perspectivas Histórica e Conceitual da Promoção de Saúde. Cogitare Enferm, $1996 ; 1(2) ; 2-7$.

12. Sícoli JL, Nascimento PR do. Promoção de saúde: concepções, princípios e operacionalização. Interface - Comunic,
Saúde, Educ, 2003; 7(12):91-112.

13. Acosta PHO de, Duarte LR. Promoção de Saúde: Promoção ou Prevenção? Rev. Fac. Ciênc. Méd. Sorocaba, 2007; 9(1):23-24.

14. Nadanovsky P. Promoção da Saúde e a Prevenção das Doenças Bucais. In: Pinto VG. Saúde Bucal Coletiva. 4. ed. São Paulo: Santos, 2000. Cap. 9, p. 293-339.

15. Campos GW, Barros RB, Castro AM de. Avaliação de política Nacional de Saúde. Ciên Saúde Colet, 2004; 9 (3):745-749.

Recebido em 08/10/2012 Aceito em 10/12/2012 